\title{
Fire detection from LEO: trade-offs for selection of spectral bands and a wide-swath optical design using MWIR and visible bands
}

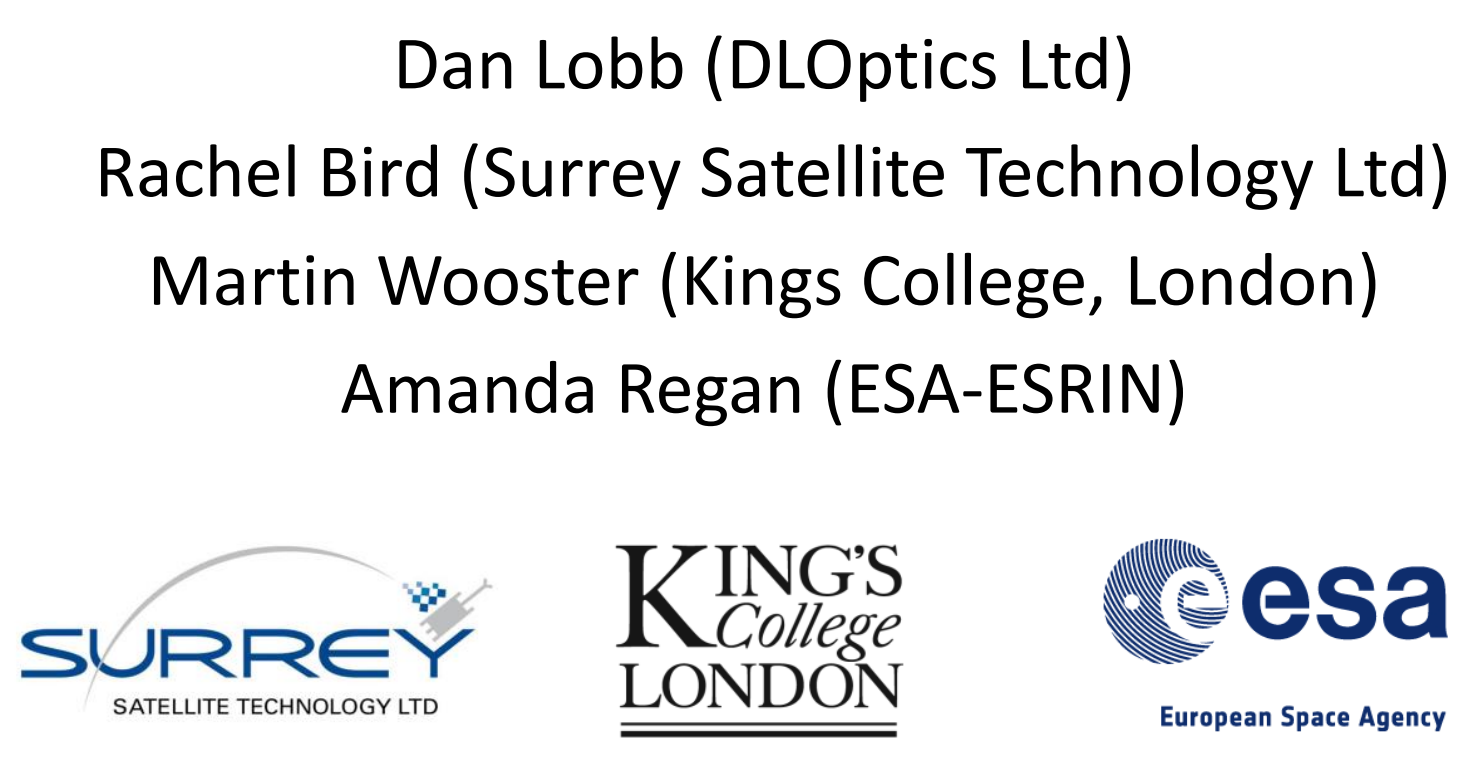




\section{Overview and background}

- The presentation addresses detection of fires from platforms in lowEarth orbit

- Logic for selection of spectral bands

- Optical design of a sensor

- Sensor performance

- Swath width

- Minimum detected Fire Radiative Power (FRP)

$>$ A main aim is to detect/measure smaller fires by providing small GSDs

- Payload requirements: envelope, mass, power, data

- Based on a study performed for ESA at SSTL

- With fire-detection analysis from Martin Wooster (KCL)

- Part of a review of possible instruments flown on platform in convoy with ESA Sentinels

- Possible complement to Sentinel 3 fire detection, using the SLSTR instrument (Sea and Land Surface Temperature Radiometer at $1 \mathrm{~km}$ GSD) 


\section{Wavebands}

- Fire detection and measurement from space has usually involved measurement of brightness temperatures (BTs) in two or more thermal wavebands

- BT is equivalent uniform black-body temperature for the measured pixel

- BT measurements are usually made in:

- At least one MWIR band ( 3 to 5 micron range) for

- Peak signal from fires and largest increase in BT from sub-pixel fires

- Best estimates of fire radiant power

- At least one LWIR band (8 to 12 micron range)

- Lower signal from fires compared with background scene variations

- Difference of BTs between MWIR and LWIR used to correct for local thermal background signal

- Fire temperatures and areas estimated using ratios of signal in MWIR and LWIR bands

- In addition, a visible/NIR (VNIR) band

- Aids discrimination against sun glint

- Generally used in cloud masking 


\section{Logic for preferring $2 * M W I R$ bands to MWIR+LWIR}

- Available MWIR detectors arrays provide more elements than LWIR arrays

- Smaller GSDs using only MWIR

- Leads to detection and measurement of smaller fires

- And/or wider swath widths

- Optics for LWIR must by a linear factor 2.5 larger than for MWIR

- if providing the same diffraction-limited GSD

- large impact on sensor mass and envelope

- MWIR detectors require less cooling than LWIR detectors

- Theoretical investigation results:

- The two selected MWIR bands will work well

- Discrimination against sun glint is more difficult for the shorter MWIR band

- But a VNIR channel to detect sun glint is desirable in any case 


\section{Relative merits of $2 * M W I R$ and MWIR+LWIR}

- Metric for detection of sub-pixel fires

- Measures Brightness Temperature Difference between two wavebands for target pixel with possible fire and

- Compares this with BTD for "background" pixels

- $\left(B T_{\text {MWIRfire }}-B T_{\text {LWIRfire }}\right)$ - $\left(B T_{\text {MWIRback }}-B T_{\text {LWIRback }}\right)$

- Plot shows comparison of the metric for

- MWIR and LWIR bands at 3900nm and 11000nm

- Compared with the equivalent for two MWIR bands at 3500nm and 4650nm

- (Wavelengths selected for relatively low uncertainty in atmosphere transmission)

- With a fairly wide separation, 2 MWIR bands appear better for lower-power sub-pixel fires

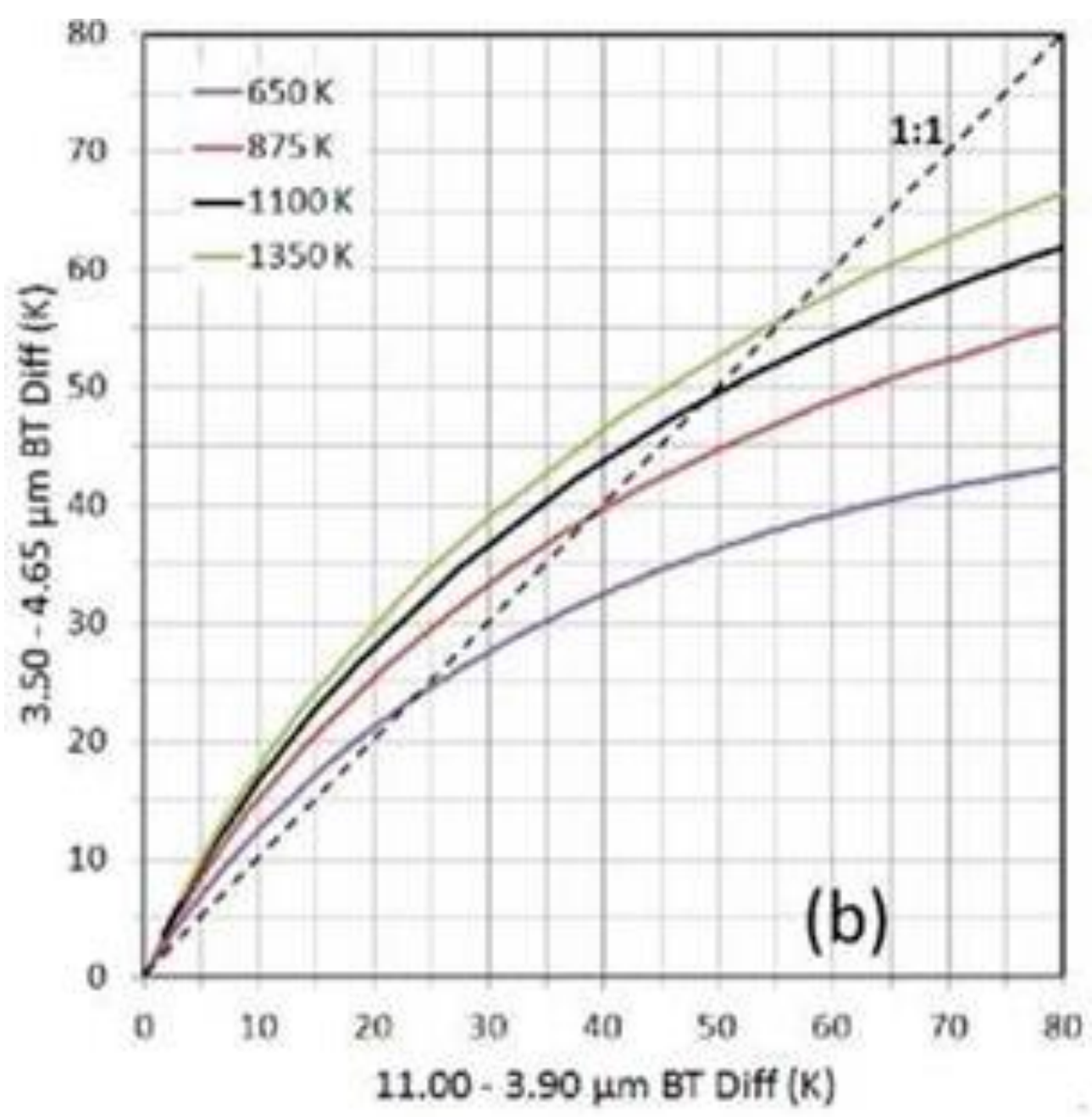




\section{MWIR detector baseline}

- Area arrays preferred (main focus for detector developments)

- Options for area-array detectors

- Each detector row will cover a section of the sensor swath width in a pushbroom imaging mode

- We can use large numbers of rows to:

- Define two (potentially more) MWIR bands using strip filters

- Multiply the total swath width

- Spatially over-sample along-track and across-track for:

- Selection of pixels with centred fires for thresholding

- Registration between spectral bands by resampling

- Enhanced SNRs for fire power/temperature assessments

- Current designs based on Selex ES SuperHawk

- $1280 \times 1024$ elements, $8 \mu \mathrm{m}$ square

- $120 \mathrm{~Hz}$ frame rate (GSD down to $60 \mathrm{~m}$ using whole frame)

- Built-in active cooling 


\section{MWIR optical design baseline}

- Swath is split between 8 telescopes using 8 sets of detector rows

- Telescopes image 8 sections of swath onto a common focal plane via fold mirrors and prisms

- Each telescope images is $60 \mathrm{~mm}$ wide ACT x (up to) $6 \mathrm{~mm}$ ALT

- Each $6 \mathrm{~mm}$ wide strip accommodates

- 2 strip filters, for bands at $3510 \mathrm{~nm}$ and $4653 \mathrm{~nm}$

- Margins for vignetting at fold optics etc

- Composite image is $60 \mathrm{~mm} \times 48 \mathrm{~mm}$

- Telescope images formed at $\mathrm{f} / 8.15$

- Relay lens

- Composite image is relayed to a single detector $10 \mathrm{~mm} \times 8 \mathrm{~mm}$

- Image on detector is formed at f/1.36

- Swath width and GSD

- Baseline gives a total of 8*1250 spatial samples ACT

- Current preferred option is $100 \mathrm{~m}$ nominal GSD

- Swath close to $1000 \mathrm{~km}$ 


\section{Baseline optics - view on nadir axis}

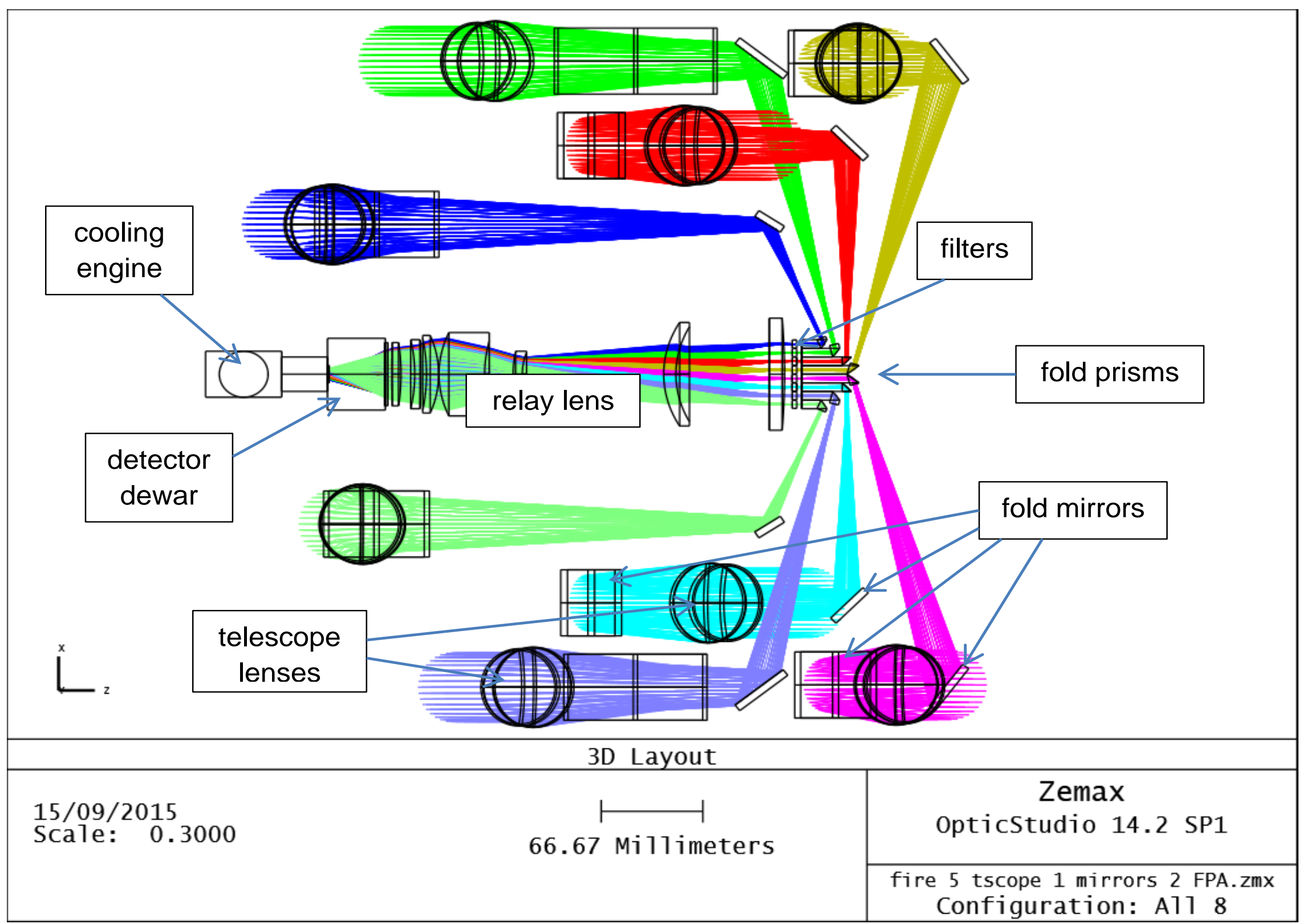




\section{Baseline optics - view along-track}

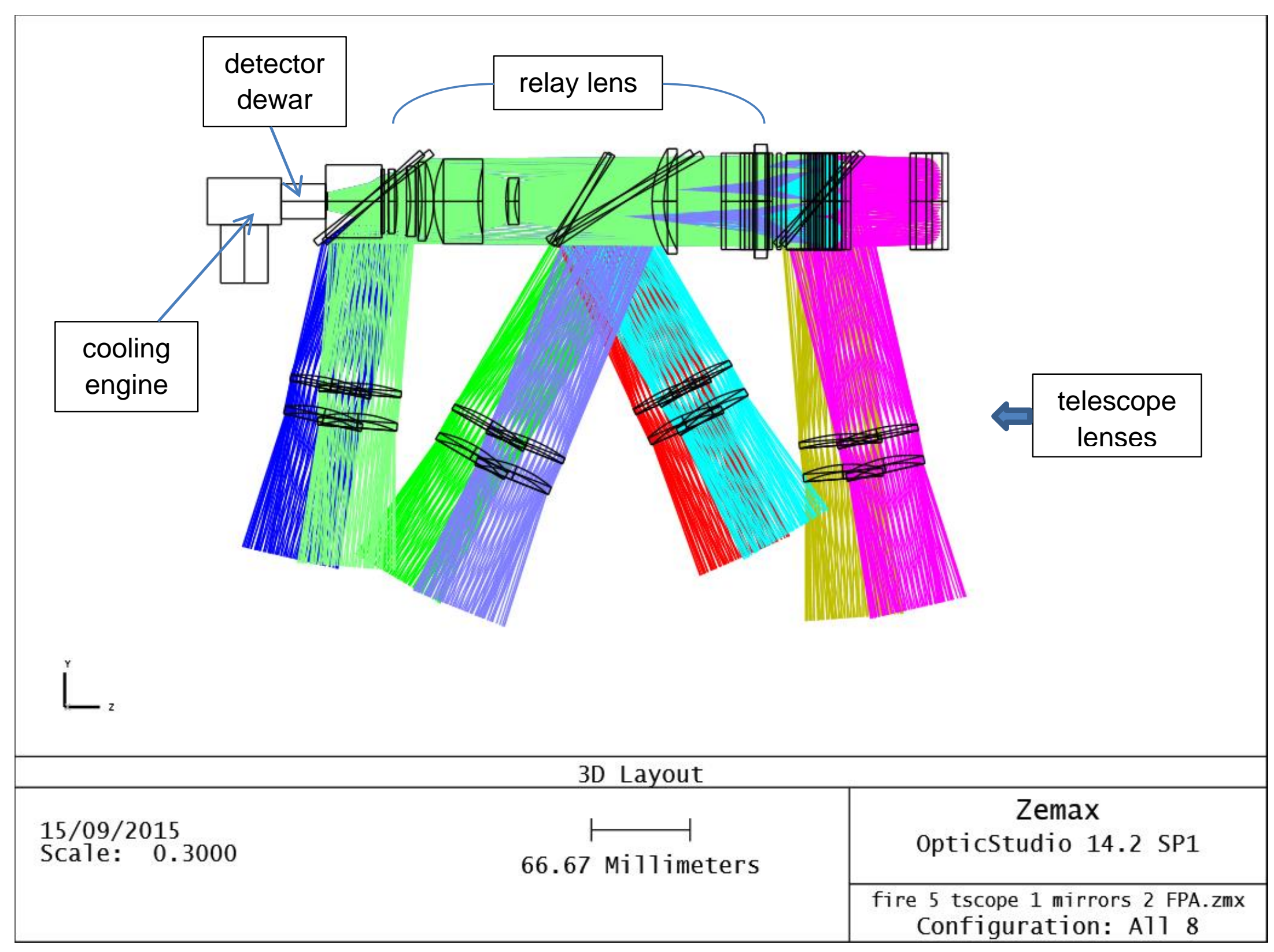




\section{Baseline optics - view across-track}

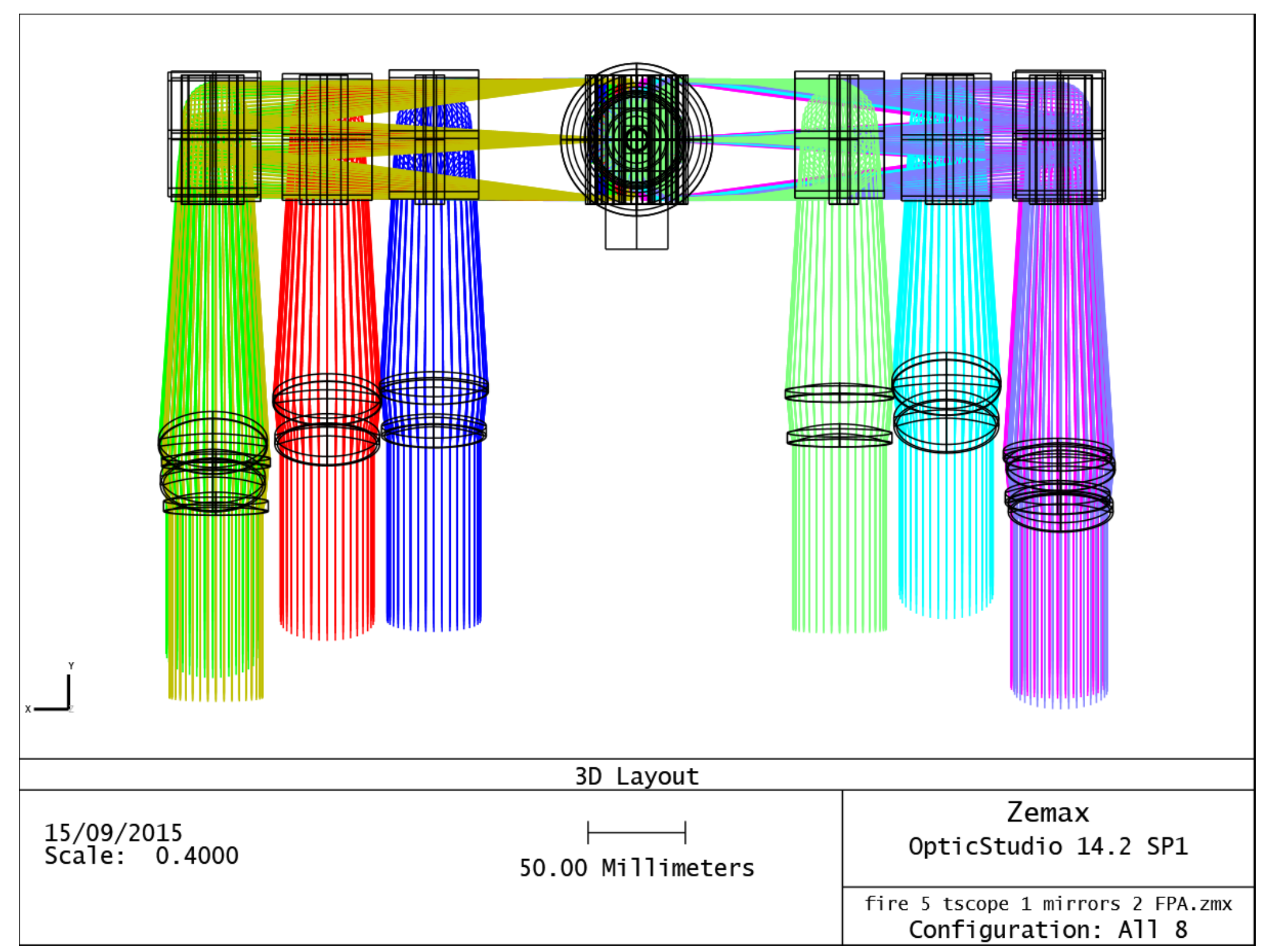


Single telescope channel

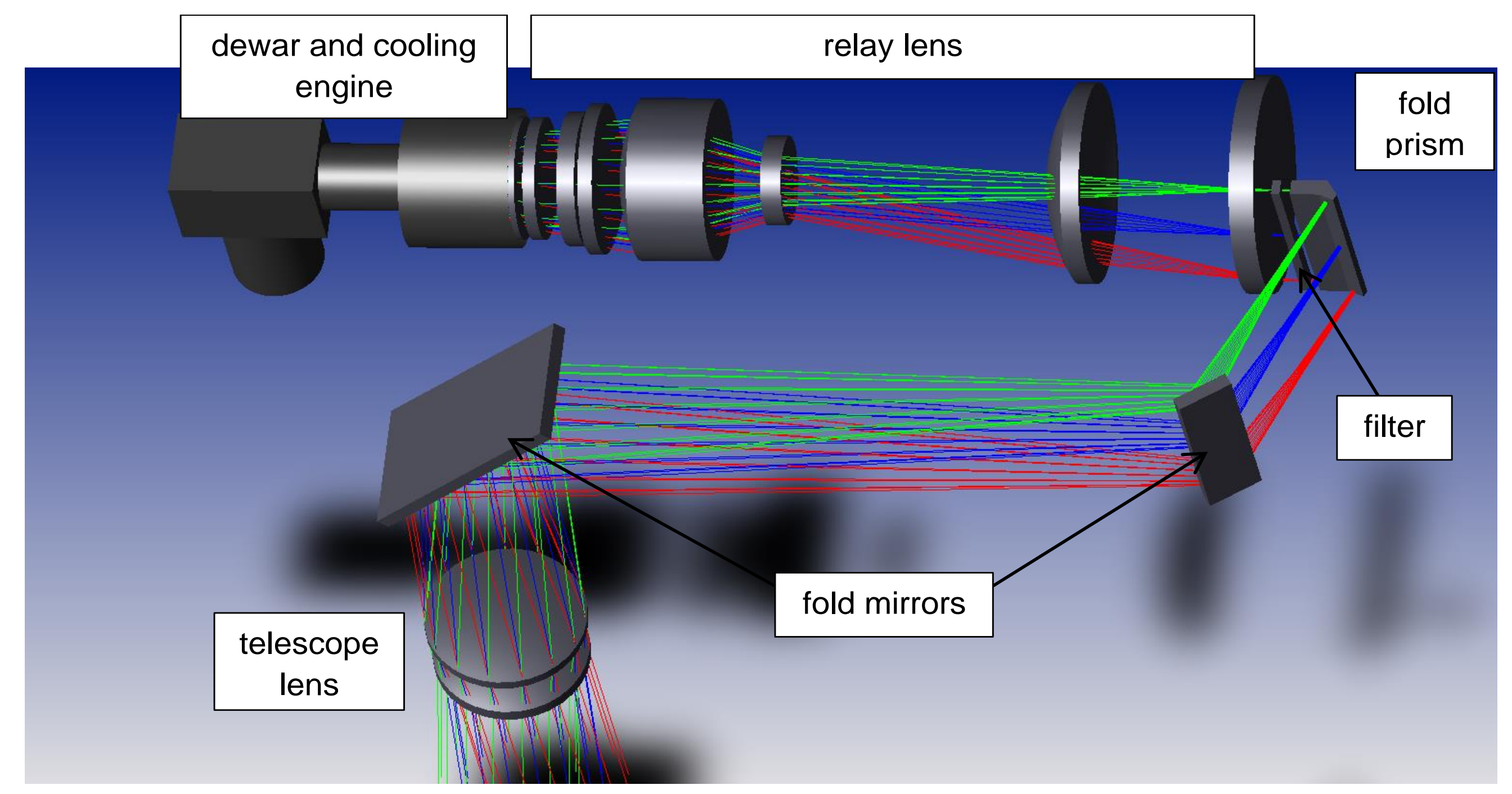


All optics perspective view

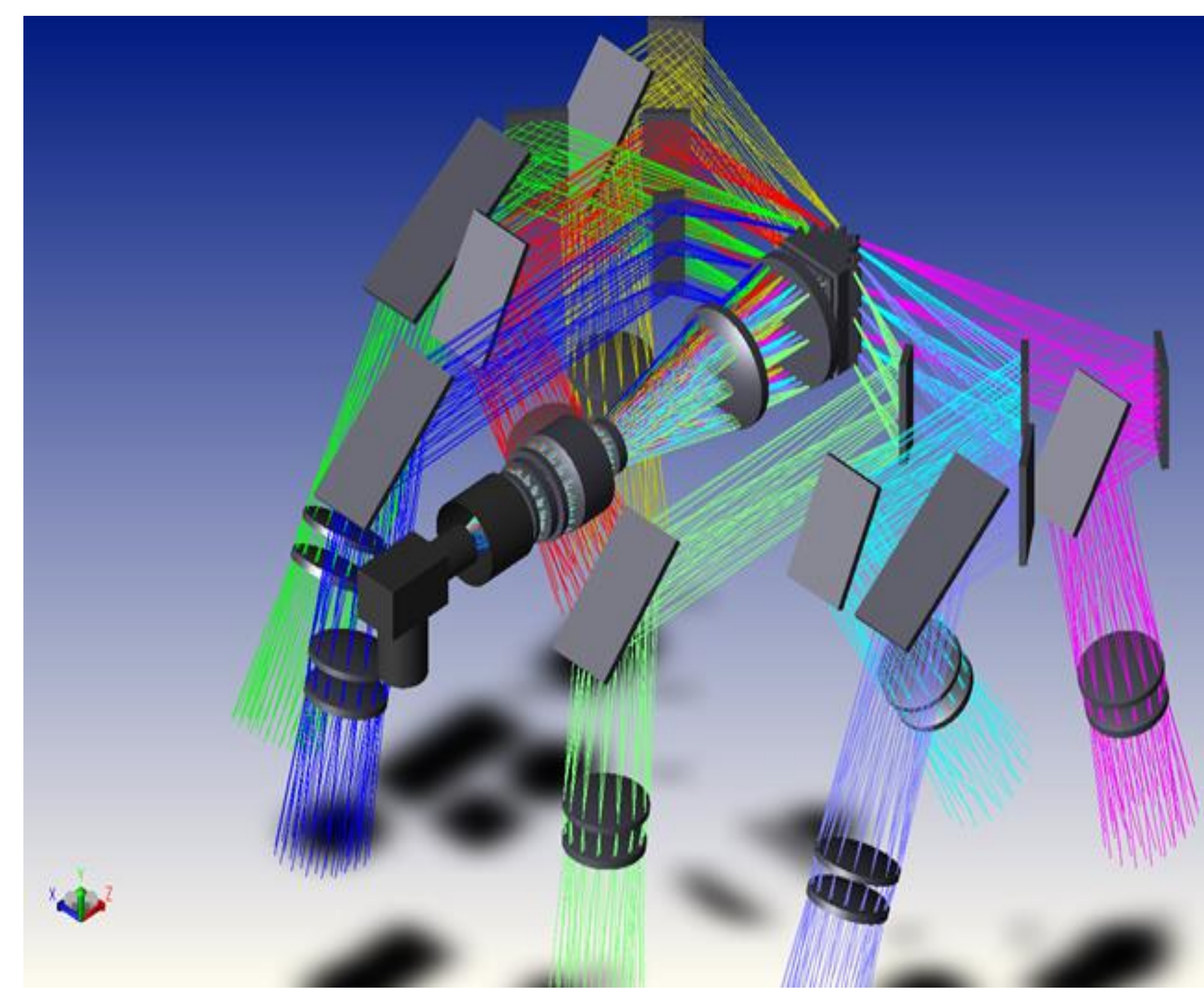


Fold prisms and filters

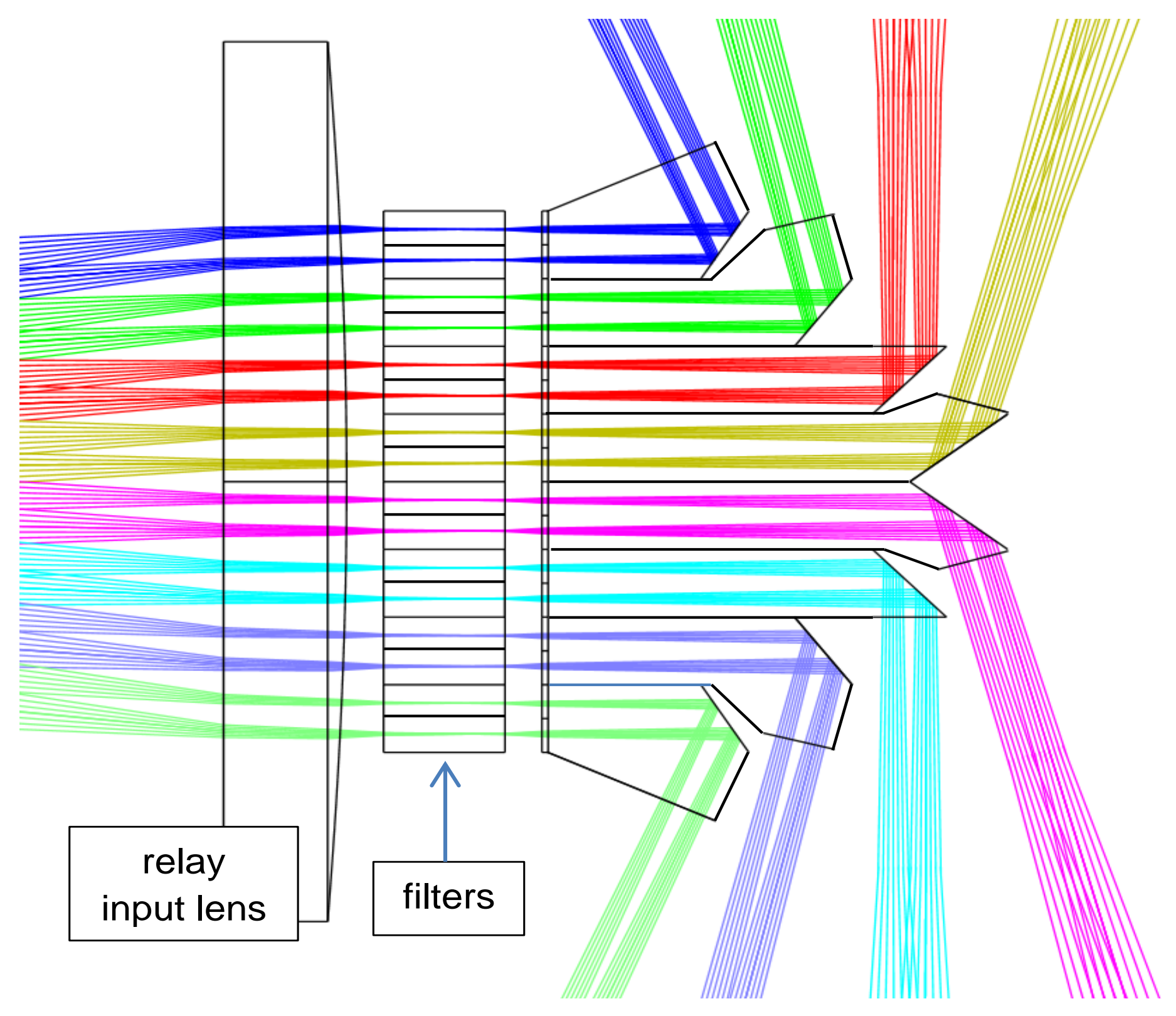




\section{Use of multiple MWIR detector rows}

- The baseline detector provides 1000 rows

- These will be split between 16 strips

- For 8 swath sections

- 2 bands in each section

- We will scan $>50$ samples/band, with margins

- These will allow factor 7 spatial over-sampling along/across track:

- Fly the platform with $1 / 49$ radians yaw

- Read detector at 8/7-times the nominal along-track sample rate

- The multiple samples will provide

- At least one centred for thresholding

- Improved SNR and accuracy with aggregation 


\section{MWIR thermal control}

- MWIR detector cooling

- Might just work at $150 \mathrm{~K}$ with passive cooling

- Active cooling $<135 \mathrm{~K}$ is preferred

- Avoids a large radiator

- May eventually allow non-sun-synchronous orbit selection

- MWIR optics

- Dewar at $<150 \mathrm{~K}$ will reduce background radiation outside an f/1.36 aperture

- Optics outside the filters

- Can be allowed temperatures up to typical scene levels

- But preferably below $270 \mathrm{~K}$

- Optics inside filters

- Need to be cooled to $<250 \mathrm{~K}$ to reduce effects of stray reflections from structures

- Strong reflection from the filters derives from the dewar 


\section{MWIR radiometric performance}

\begin{tabular}{|l|c|}
\hline \multicolumn{1}{|c|}{ Parameter } & Value \\
\hline GSD at altitude 814km & $100 \mathrm{~m}$ \\
\hline Dwell period (adjusted to de-synchronise) & $13 \mathrm{~ms}$ \\
\hline Aperture diameter & $48 \mathrm{~mm}$ \\
\hline Focal length & $65 \mathrm{~mm}$ \\
\hline Detector element size & $8 \mathrm{\mu m}$ \\
\hline Spectral band 1 & $3510 \mathrm{~nm} \pm 20 \mathrm{~nm}$ \\
\hline Spectral band 2 & $4653 \mathrm{~nm} \pm 20 \mathrm{~nm}$ \\
\hline Maximum fire temperature & $1350 \mathrm{~K}$ \\
\hline Minimum fire temperature & $650 \mathrm{~K}$ \\
\hline Optics transmission from scene & $50 \%$ \\
\hline Quantum efficiency & $80 \%$ \\
\hline Read noise & $400 \mathrm{e}-\mathrm{rms}$ \\
\hline Detector temperature & $120 \mathrm{~K}$ \\
\hline Cool structure (after filters) temperature & $250 \mathrm{~K}$ \\
\hline Dewar temperature & $150 \mathrm{~K}$ \\
\hline Detector cut-off wavelength & $5000 \mathrm{~nm}$ \\
\hline \multicolumn{1}{|c|}{ Results } & $480 \mathrm{mK}$ \\
\hline NEdT for scene at 300K, single pixel - band 1 & $90 \mathrm{mK}$ \\
\hline NEdT for scene at 300K, single pixel - band 2 \\
\hline
\end{tabular}




\section{MWIR spatial performance}

- Spot diagrams show

- $8 \mu \mathrm{m}$ element size

- Airy diameter for $4653 \mathrm{~nm}$

- Enclosed energy is limited by diffraction

- $82 \%$ at $3150 \mathrm{~nm}$

- $67 \%$ at $4653 \mathrm{~nm}$

- $58 \%$ with motion smearing

- Effects of spatial resolution

- For NEdTs: not significant compared with background uncertainties

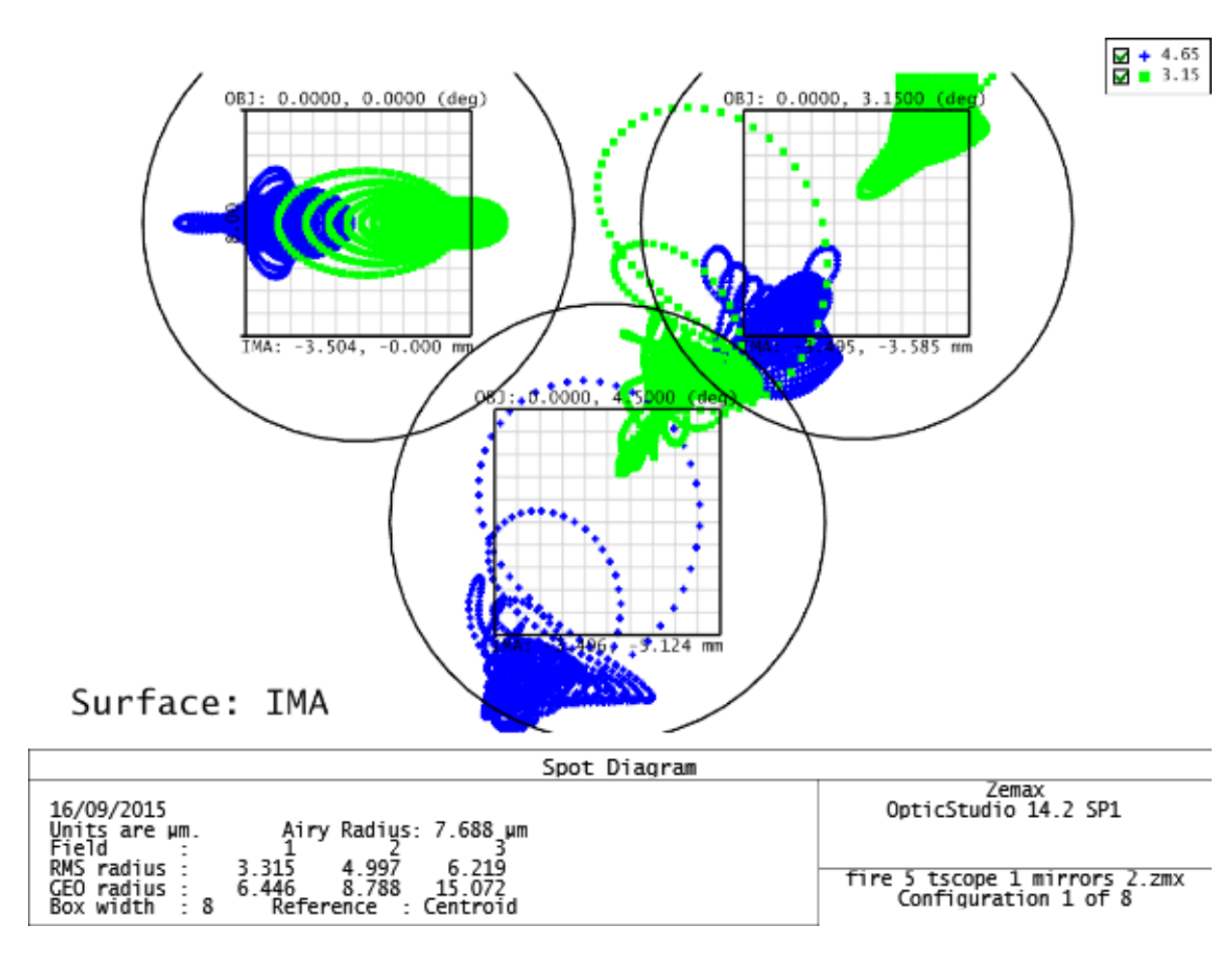

- Threshold levels can be adjusted for known losses 


\section{Ghost imaging and dichroic option}

- Ghost imaging to increase dynamic range

- Uncoated ZnS wedge outside each telescope (0.1 $1^{\circ}$ angle)

- Introduce a set of multiple images separated along-track

- Relative intensities 100, 2.2, 0.048...

- Allows imaging within saturation of very large fires

- Dichroic to superimpose MWIR bands

- Baseline gives bands separated along-track $0.32(4.6 \mathrm{~km})$

- A dichroic and fold mirror can superimpose images

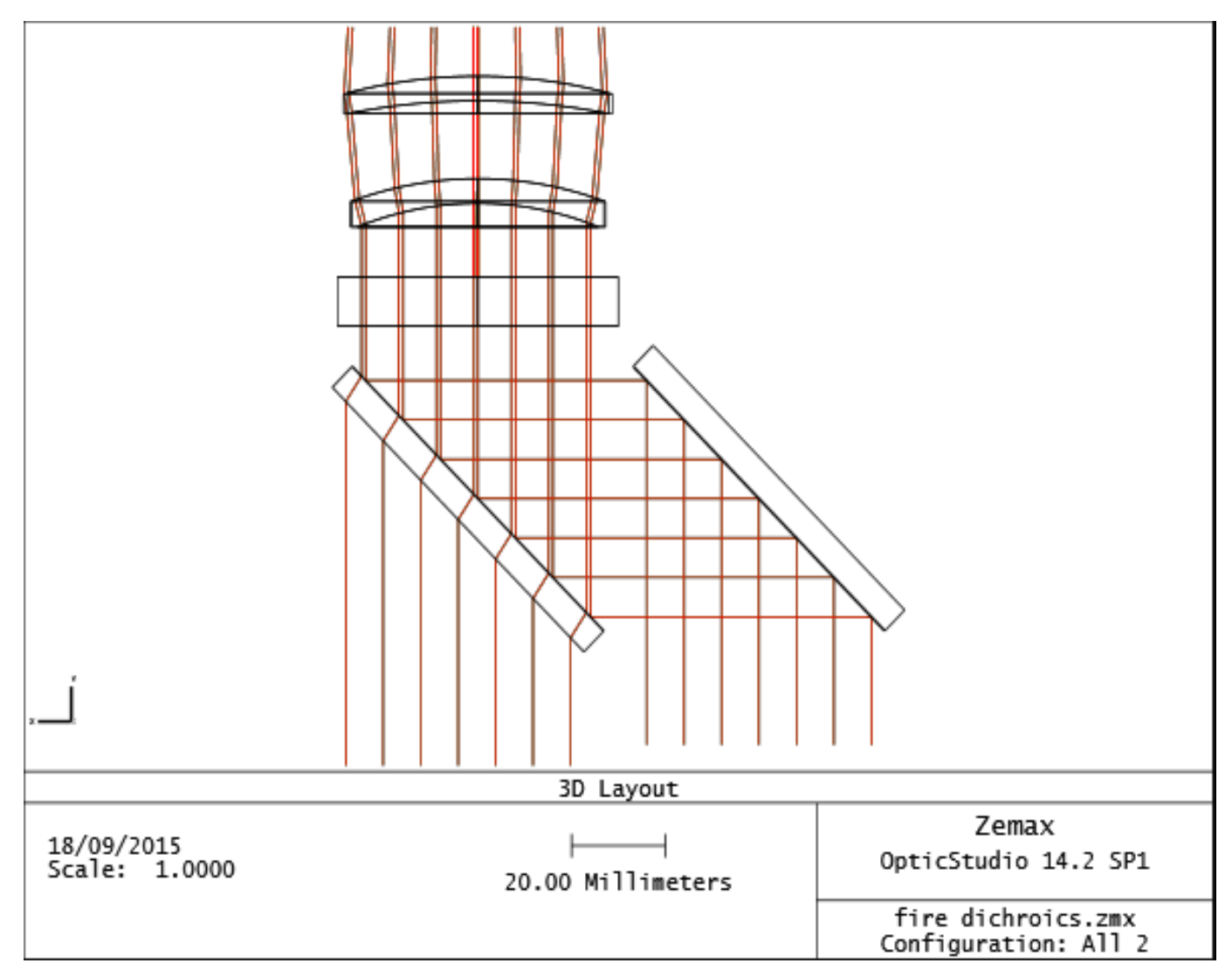

- Not necessarily the baseline 


\section{VNIR channel design}

- Required critically to reject false-detections due to sun-glint:

- Any VNIR band may be useable

- Registered with (at least) one MWIR band to a fraction of sun-subtense

- Should be spatially over-sampled across-track with respect to MWIR to allow effective registration

- May be used for cloud mapping (if not provided by a convoy partner)

- Baseline is a simple pushbroom system using linear array detectors

- 39m GSD, using 2 x 12,000-element ( $8 \mu \mathrm{m}$ ) arrays (e2v CCD 21-40)

- 2 lenses with $167 \mathrm{~mm}$ focal length cover $1000-\mathrm{km}$ swath $\left(2 \times 32^{\circ}\right.$ fields)

- Nominal aperture diameter: $20 \mathrm{~mm}$

- Nominal spectral band $500 \mathrm{~nm}$ to $600 \mathrm{~nm}$ 


\section{VNIR optics}

- VNIR imaging

- Using two linear arrays

- Separate lenses

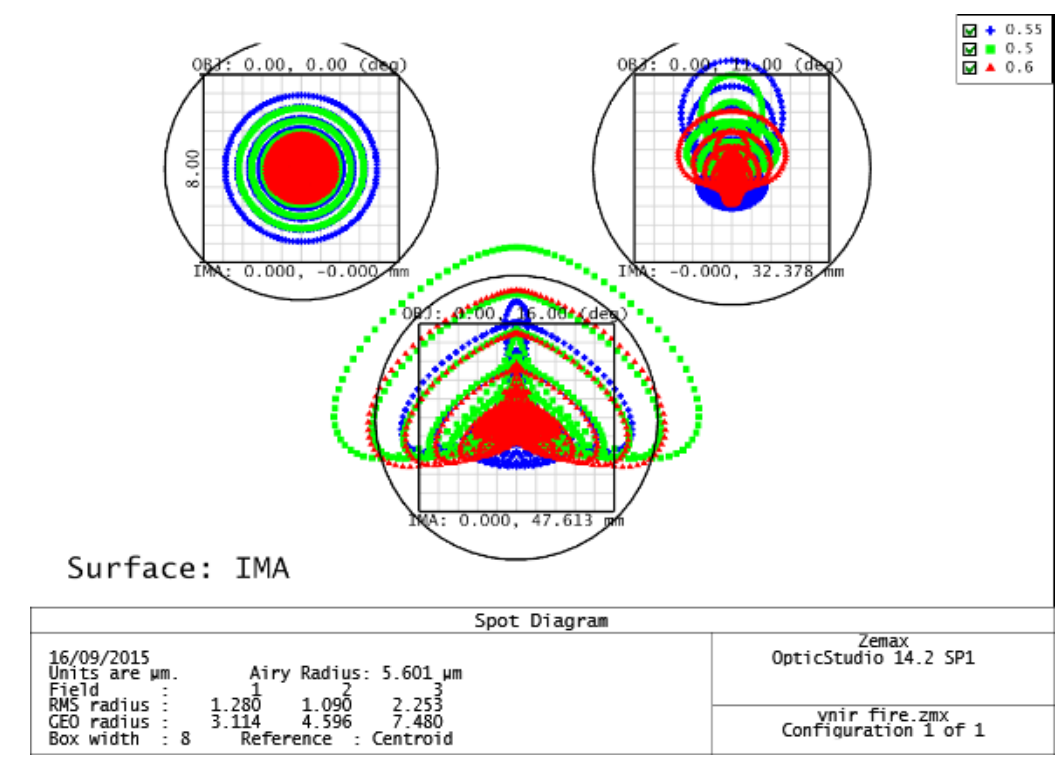

DLOptics

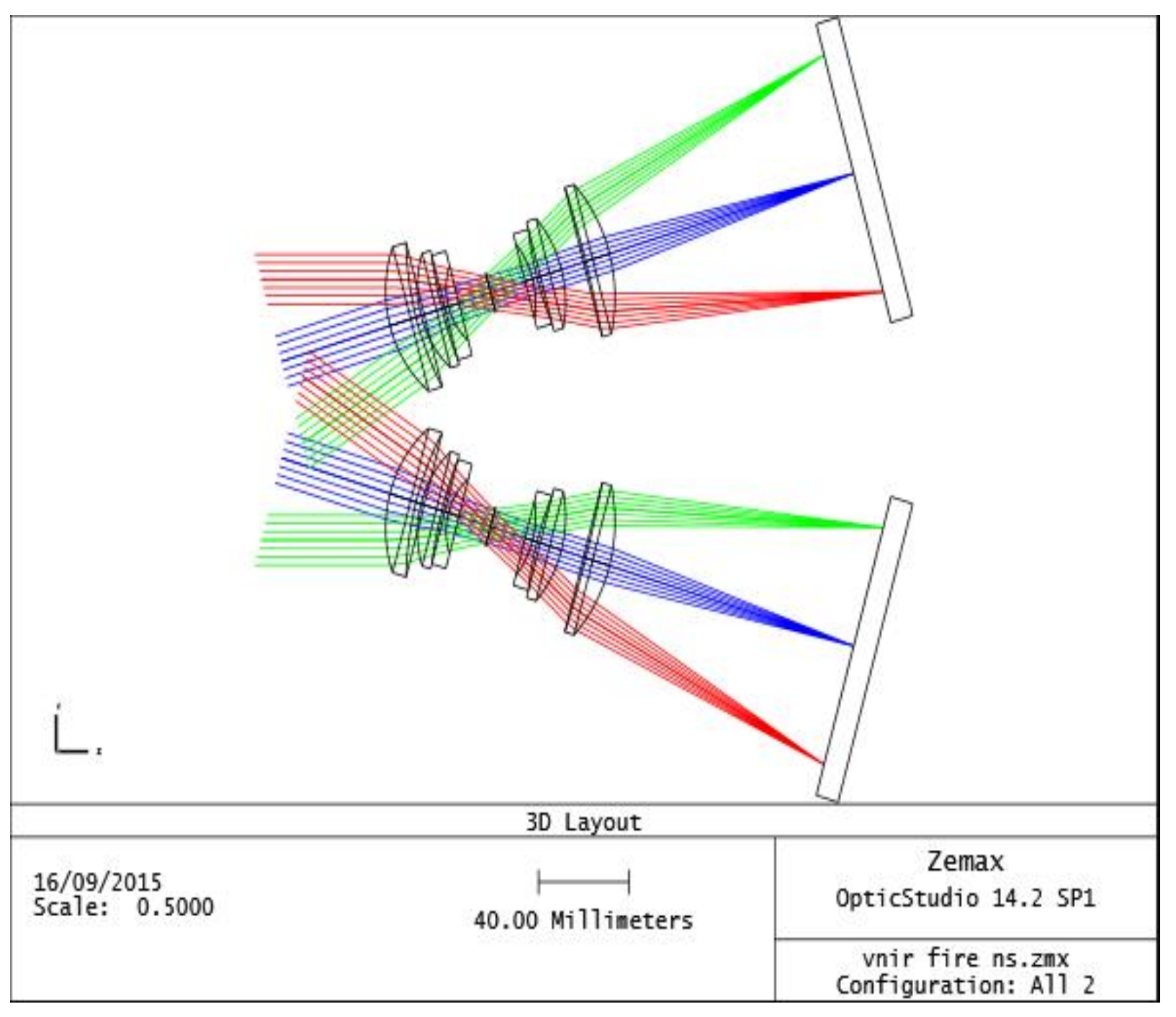




\section{VNIR channel analysis}

\begin{tabular}{|l|c|}
\hline \multicolumn{1}{|c|}{ Parameter } & Value \\
\hline GSD at altitude 814km & $39 \mathrm{~m}$ \\
\hline Dwell period with x2 along-track over-sampling & $3.0 \mathrm{~ms}$ \\
\hline Aperture diameter & $20 \mathrm{~mm}$ \\
\hline Focal length & $167 \mathrm{~mm}$ \\
\hline Centre of spectral band & $550 \mathrm{~nm}$ \\
\hline Band width & $100 \mathrm{~nm}$ \\
\hline Solar irradiance in band & $1800 \mathrm{~W} \cdot \mathrm{m}^{-2} \cdot \mathrm{mm}^{-1}$ \\
\hline Optics transmission & $90 \%$ \\
\hline Quantum efficiency & $50 \%$ \\
\hline Read noise & $40 \mathrm{e}-\mathrm{rms}$ \\
\hline Digitisation & $12 \mathrm{bits}$ \\
\hline "Maximum" albedo & $120 \%$ \\
\hline "Reference" albedo & $20 \%$ \\
\hline Detector saturation level & $400,000 \mathrm{e}-$ \\
\hline & \\
\hline Signal \% of saturation at maximum albedo & $12 \%$ \\
\hline SNR at reference albedo (single sample) & 75 \\
\hline Raw data rate & $4.2 \mathrm{Mpixel} / \mathrm{s}$ \\
\hline
\end{tabular}




\section{Fire detection capability (simplified)}

- Sun glint threshold for daylight operation

- Provisionally we set a rejection threshold at $100 \%$ diffuse equivalent VNIR reflectance

- Equivalent to $18 \mathrm{~K}$ delta brightness temperature at $300 \mathrm{~K}$, in the $4653 \mathrm{~nm}$ band

- MWIR Brightness Temperature threshold could be set at $\sim 25 \mathrm{~K}$ allowing for some background variation

- Fire Fractional Area 0.0046 for a fire at $650 \mathrm{~K}$ with $300 \mathrm{~K}$ background

- Fire Fractional Area 0.00036 for a fire at $1350 \mathrm{~K}$

- Fire radiative power in $100 \mathrm{~m}$ square pixel:

- $0.45 \mathrm{MW}$ at $650 \mathrm{~K}$

- $0.65 \mathrm{MW}$ at $1350 \mathrm{~K}$

- Fire temperature estimates and fractional areas will be refined using ratios of signal in $2 \mathrm{MWIR}$ bands 


\section{Fire payload budgets}

- Envelope

- Across-track: $600 \mathrm{~mm}$

- Along-track: $700 \mathrm{~mm}$

- Nadir axis: $300 \mathrm{~mm}$

- Mass: $50 \mathrm{kgm}$

- Power: 35W

- Raw data rate

- MWIR: 63Mpixels/s

- VNIR: 3.8Mpixels/s

- Data recorded per orbit

- For 1000 fires

- $16 \mathrm{~km}^{2}$ around each fire

- At 14 bits

- 1120 Gbits
MWIR detector

MWIR detector cooler

MWIR electronics

MWIR optics cooler

VNIR detectors FEE

Temperature control

Total

\section{MWIR raw data rate}

VNIR raw data rate

MWIR rows recorded per fire

MWIR columns recorded per fire

MWIR frames recorded per fire

VNIR rows recorded per fire

VNIR columns recorded per fire

Bits recorded

MWIR data recorded per fire

VNIR data recorded per fire

Number of potential fires/orbit

Total data recorded per orbit

$\begin{array}{rl}2.5 & \text { W } \\ 10 & W \\ 5 & W \\ 5 & W \\ 10 & W \\ 2.5 & W \\ 35 & W\end{array}$

1120 Gbits 


\section{Conclusions on fire payload}

- There is a strong case for limiting the thermal IR bands to mid-wave - a VNIR channel is also needed

- Existing MWIR detectors provide large numbers of elements allowing small GSDs and large swath

- A baseline design is suggested which is likely to provide

- $1000 \mathrm{~km}$ swath width

- Identification of fires down to FRPs around 0.5MW in $100 \mathrm{~m}$ ground samples

- More work is needed to investigate substantial uncertainties affecting performance - e.g.:

- Variations in atmosphere spectral transmission

- Uncertainties in correction for background temperature variations

- Uncertainties in spectral emissivities and solar reflectances

- Design is likely to change substantially on optimisation 


\section{Thanks for your attention (Easy) questions please}

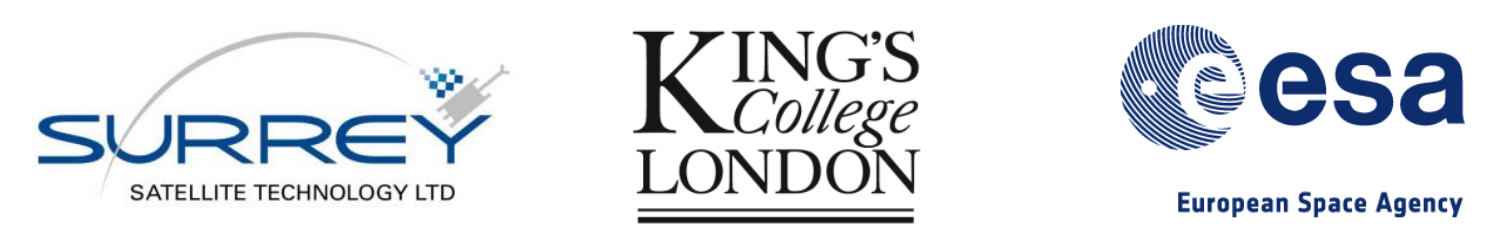

